\title{
Restoration of Isospin Symmetry in Highly Excited Nuclei
}

\author{
H. Sagawa ${ }^{1}$, P.F. Bortignon ${ }^{2}$ and G. Colò ${ }^{2}$ \\ 1 Center for Mathematical Sciences, University of Aizu, Aizu-Wakamatsu, Fukushima 965, Japan \\ ${ }^{2}$ Dipartimento di Fisica, Università degli Studi and INFN, Sezione di Milano, Via Celoria 16, \\ 20133 Milano, Italy
}

(October 20, 2018)

\begin{abstract}
Explicit relations between the isospin mixing probability, the spreading width $\Gamma_{I A S}^{\downarrow}$ of the Isobaric Analog State (IAS) and the statistical decay width $\Gamma_{c}$ of the compound nucleus at finite excitation energy, are derived by using the Feshbach projection formalism. The temperature dependence of the isospin mixing probability is discussed quantitatively for the first time by using the values of $\Gamma_{I A S}^{\downarrow}$ and of $\Gamma_{c}$ calculated by means of microscopic models. It is shown that the mixing probability remains essentially constant up to a temperature of the order of $1 \mathrm{MeV}$ and then decreases to about $1 / 4$ of its zero temperature value, at higher temperature than $\approx 3 \mathrm{MeV}$, due to the short decay time of the compound system.
\end{abstract}

PACS and Keywords: 21.10.Hw, 25.70.Gh, 24.30.Cz; isotopic spin, symmetry restoration, compound nucleus. 
Isospin symmetry is the first dynamical symmetry in nuclear physics proposed by W. Heisenberg in 1932 [1] and has played a very important role in the study of the structure of atomic nuclei for many years. One of the highlight of the isospin symmetry is the discovery of the isobaric analog state (IAS) of the parent ground state in the daughter nucleus by means of charge exchange reactions [2]. Recently, problems related to the isospin symmetry and its breaking have been discussed with new emphasis because new experiments in nuclei near the drip lines have become possible. These radioactive beams facilities can provide access to regions of large isospin deformations [3]. Several theoretical predictions have been made for the isospin mixing and large mixing probabilities in heavier nuclei near the proton drip line are found, up to $(5-6) \%$ in ${ }^{100} \mathrm{Sn}$ 迎. Possible effects of these large isospin impurity are at present under consideration, both by experimentalists and theoreticians, in connection with the width of the IAS and the Fermi $\beta^{-}$decay of $\mathrm{N} \cong \mathrm{Z}$ nuclei.

A restoration of the isospin symmetry in highly excited compound nuclei was pointed out by H. Morinaga [5] and D.H. Wilkinson [6] already 40 years ago. This problem has been studied experimentally by measuring the cross sections for the de-excitation of the giant dipole resonances (GDR) in thermal, self-conjugate nuclei. The experimental data show the sharp decrease of the mixing amplitude at high excitation energies above $E^{*}=20 \mathrm{MeV}$ in ${ }^{28} \mathrm{Si}$ and ${ }^{60} \mathrm{Zn}$ [7] as well as in ${ }^{40} \mathrm{Ca}$ [8]. The restoration of the isospin symmetry was theoretically discussed by using a simple model in Ref. [9].

In this letter, we would like to address the problem of the restoration of the isospin symmetry by using a microscopic model, which was recently proposed and used at zero temperature in Ref. [10]. This model is based on the Feshbach projection method and gives an explicit relation between the spreading width of the IAS and the isospin mixing probability. In Ref. [10] this relation was employed successfully to calculate, from the values of the isospin mixing, the spreading widths of the IAS in Sn- and I-isotopes and in ${ }^{208} \mathrm{Bi}$. In the present work, we will extend the microscopic model to the case of excited compound nuclei, and study quantitatively the temperature dependence of the isospin mixing through its relation with the spreading width of the IAS. 
The isospin mixing amplitude is written in perturbation theory as [1]

$$
\alpha^{T_{0}+1}=\frac{1}{E^{T_{0}}-E_{M}^{T_{0}+1}}\left\langle M ; T=T_{0}+1, T_{z}=T_{0}\left|H_{1}\right| T=T_{0}, T_{z}=T_{0}\right\rangle,
$$

where $H_{1}$ includes the isospin non-conserving interactions, i.e., the isovector part of the Coulomb interaction and the charge symmetry breaking as well as charge independence breaking interactions. The state with isospin $T_{0}$ is the mother state (either the ground state or the IAS), while the states with isospin $T_{0}+1$ give rise to the isospin impurity in the mother state by coupling to it. In the cases of medium-heavy and heavy nuclei, the isovector giant monopole resonances (IVGMR) give the major contribution to the isospin mixing so that we consider only the coupling to these states in the following.

At finite temperature the mother state lies at some high excitation energy and its lifetime as well as that of the IVGMR built on top of it, are affected by statistical emission of particles (mainly neutrons) and $\gamma$-rays. The corresponding width is the compound width $\Gamma_{c}$ and the denominator of Eq. (11) should contain the sum of the imaginary terms $i \Gamma_{c}^{T_{0}} / 2$ and $i \Gamma_{c}^{T_{0}+1} / 2$ [12]. We simply denote in the following this sum as $i \tilde{\Gamma}_{c} / 2$ and $\tilde{\Gamma}_{c}$ will be called "compound width". In general, we put a tilde over the widths at finite temperature. The compound width will be added to the sum $\tilde{\Gamma}_{M}$ of the intrinsic escape and spreading widths of the $T_{0}+1$ monopole state. We discuss later how all these widths are estimated by using microscopic models.

Let us now study the relation between the isospin mixing amplitude and the spreading width of the IAS. We will use the same Feshbach projection method of Ref. [10] by extending it to the case of excited compound nuclei. The whole model space of nuclear configurations is built up with states which are eigenstates of the isospin-conserving nuclear Hamiltonian $H_{0}$ and is divided in two subspaces $\mathrm{P}$ and $\mathrm{Q}$. The $\mathrm{P}$ space contains only two states, the parent ground state and the IAS which have isospin $T_{0}$. The other nuclear states in the daughter nucleus which have isospin $T_{0}-1$ form the space Q. The spreading width of the IAS $\left(\tilde{\Gamma}_{I A S}^{\downarrow}\right)$ is obtained by coupling with doorway states of the space $\mathrm{Q}$ through the isospin-breaking Hamiltonian as 10 


$$
\tilde{\Gamma}_{I A S}^{\downarrow}\left(E^{*}\right)=-2 \operatorname{Im}\left\langle\mathrm{IAS}\left|H_{1} Q \frac{1}{E^{*}-H_{Q Q}} Q H_{1}\right| \mathrm{IAS}\right\rangle
$$

where $E^{*}$ is the energy of the IAS in the compound nucleus and $H_{Q Q}$ is the matrix element of the isospin-conserving Hamiltonian $H_{0}$ between states in Q-space, among which the dominant role is played by the three isospin components of the IVGMR in the daughter nucleus. At finite temperature, both the states in P- and Q-spaces have finite decay times, as discussed above. In terms of $\tilde{\Gamma}_{c}$ and $\tilde{\Gamma}_{M}$, Eq. (2) is rewritten as

$$
\begin{aligned}
\tilde{\Gamma}_{I A S}^{\downarrow} & =-2 \operatorname{Im} \sum_{q} \frac{\left|\left\langle\mathrm{IAS}\left|H_{1}\right| q\right\rangle\right|^{2}}{E^{*}-E_{q}+i \tilde{\Gamma}_{c}\left(E^{*}\right) / 2+i \tilde{\Gamma}_{M}\left(E^{*}\right) / 2} \\
& =\sum_{q}\left(\tilde{\Gamma}_{c}\left(E^{*}\right)+\tilde{\Gamma}_{M}\left(E^{*}\right)\right) \frac{\left|\left\langle\mathrm{IAS}\left|H_{1}\right| q\right\rangle\right|^{2}}{\left(E^{*}-E_{q}\right)^{2}+\left(\tilde{\Gamma}_{c}\left(E^{*}\right) / 2+\tilde{\Gamma}_{M}\left(E^{*}\right) / 2\right)^{2}}
\end{aligned}
$$

where the $E_{q}$ are the excitation energies of the three different isospin components of the IVGMR in daughter nuclei, which have $T^{\prime}=T-1, T$ and $T+1$ and $\tilde{\Gamma}_{c}\left(E^{*}\right)$ means, as recalled above, the sum of the two compound widths at the excitation energy corresponding to the nulear temperature, and at that energy plus the IAS energy $E^{*}$. The isospin structure of the IVGMR can be treated properly by the Feshbach projection method as it has been done in Ref. [10]. The right hand side of Eq. (3) results as a product of three factors, by assuming that for $\tilde{\Gamma}_{c}$ and $\tilde{\Gamma}_{M}$ average values over $q$ can be taken, and precisely

$$
\tilde{\Gamma}_{I A S}^{\downarrow}=\left(\tilde{\Gamma}_{c}\left(E^{*}\right)+\tilde{\Gamma}_{M}\left(E^{*}\right)\right)\left(\alpha^{T_{0}+1}\right)^{2}\left(T_{0}+1\right) F\left(T_{0}\right),
$$

where the isospin factor $F(T)$ is given by

$F(T)=\frac{1}{T}\left\{\left(\frac{2 T-1}{2 T+1}\right) \frac{1}{\left(\Delta E_{M}^{T-1}\right)^{2}}+\frac{(T-1)^{2}}{T+1} \frac{1}{\left(\Delta E_{M}^{T}\right)^{2}}+\frac{4 T^{2}}{(2 T+1)(T+1)} \frac{1}{\left(\Delta E_{M}^{T+1}\right)^{2}}\right\}\left(\Delta E_{M \pi}\right)^{2}$.

In this last Equation, $\Delta E_{M}^{T^{\prime}}=E_{M}^{T^{\prime}}-E^{*}$ is the energy difference between the three isospin components of the IVGMR in the daughter nucleus and the energy of the IAS whereas $\Delta E_{M \pi}=E_{M}^{T+1}-E_{\pi}$ is the energy difference between the $T+1$ component of the IVGMR in the mother nucleus and the mother state $|\pi\rangle$. This last difference enters because the isospin mixing amplitude $\alpha^{T_{0}+1}$ is obtained by Eq. (11). In Ref. [10 the isospin splitting was taken 
care of by using the Lane potential and we write a similar expression for nuclei at finite temperature,

$$
\Delta E_{M}^{T^{\prime}}=E^{*}(I V G M R)+\frac{V_{1}}{A} \mathbf{t} \cdot \mathbf{T}_{\mathbf{c}}
$$

where $E^{*}(I V G M R)$ is the average energy of the IVGMR in the compound nucleus and $\mathbf{t}\left(\mathbf{T}_{\mathbf{c}}\right)$ is the isospin of the IVGMR (the daughter nucleus). The value $E^{*}(I V G M R)$ is taken from the systematics in nuclei at zero temperature as

$$
E^{*}(I V G M R)=170 A^{-1 / 3} \mathrm{MeV} .
$$

The value of the parameter of the Lane potential commonly used is $V_{1}=120 \mathrm{MeV}$. In general, the Brink-Axel hypothesis suggests that giant resonances built on excited states lie at the same energy as the corresponding resonances built on the ground state. In particular, in the case of the isovector giant dipole resonance it has been experimentally observed that the excitation energy does not vary appreciably with increasing temperature [13]. Thus, it is expected that the energy of the IVGMR also does not vary with increasing temperature.

The isospin geometrical factor $F\left(T_{0}\right)$ is certainly important to predict a proper isotope dependence of the spreading width $\tilde{\Gamma}_{I A S}^{\downarrow}$ of several nuclei in medium-mass region. For our present purpose, however, we may be allowed to discard the isospin dependence of the IVGMR energies. Then, Eq. (四) becomes

$$
\tilde{\Gamma}_{I A S}^{\downarrow} \cong\left(\tilde{\Gamma}_{c}\left(E^{*}\right)+\tilde{\Gamma}_{M}\left(E^{*}\right)\right)\left(\alpha^{T_{0}+1}\right)^{2}\left(T_{0}+1\right)
$$

so that the isospin mixing probability is given by

$$
\left(\alpha^{T_{0}+1}\right)^{2}=\frac{1}{T_{0}+1} \frac{\tilde{\Gamma}_{I A S}^{\downarrow}\left(E^{*}\right)}{\tilde{\Gamma}_{c}\left(E^{*}\right)+\tilde{\Gamma}_{M}\left(E^{*}\right)} .
$$

In Eq. (9), $\tilde{\Gamma}_{c}$ is expected to rise significantly with temperature. On the other hand, the values of the spreading width $\tilde{\Gamma}_{I A S}^{\downarrow}$ and of the total width $\tilde{\Gamma}_{M}$ are not expected to be very much temperature dependent, because of the following reasons. The temperature dependence of the intrinsic escape widths $\tilde{\Gamma}^{\uparrow}$ of giant resonances is discussed in Ref. 14 and is found to 
be small. For the intrinsic spreading widths $\tilde{\Gamma}^{\downarrow}$, the authors of Ref. [15 predict a very mild temperature dependence. Consequently, from Eq. (9) we should expect at high temperature a strong decrease of the isospin mixing, or equivalently a restoration of the isospin symmetry. Eq. (9) certainly justifies the conjecture which was raised by Wilkinson [6] to describe the isospin mixing problem: if the compound nucleus decays on a time scale which is shorter than the time needed for a well-defined isospin state to mix with states with different isospin, then the isospin symmetry is partially or totally restored. Consequently, the restoration of the isospin symmetry can be understood on the ground of simple kinetic arguments (as pointed out also in Ref. [9]). The same kinetic arguments are used in Ref. [16], where an equation with the same physical content as our Eq. (9) is obtained within the context of the study of the disappearance of the collective giant resonance strength at high excitation energy.

The isospin mixing in compound nuclei was also discussed extensively in Ref. [17]. The authors derived a formula for the isospin mixing probability $\alpha^{2}$ in self-conjugate nuclei as

$$
\left(\alpha^{T_{0}+1}\right)^{2}=\frac{\Gamma_{2}^{\downarrow}}{\Gamma_{2}^{\uparrow}},
$$

where $\Gamma_{2}^{\downarrow}$ and $\Gamma_{2}^{\uparrow}$ are the mixing and total decay width of the states in the channel 2 which is equivalent to the Q-space of our model. Although they do not specify the spin and parity of the states in channel 2, the formula (10) looks similar to our Eq. (9) except for the isospin factor which is necessary for non self-conjugate nuclei. It should be noticed that the two models give almost the same final result, although the formalisms by us and by the authors of [17] look at first sight very different.

To discuss quantitatively the dependence of the isospin mixing on the excitation energy of the compound nucleus, in the example of ${ }^{208} \mathrm{~Pb}$, we have to model the temperature dependence of all quantities which appear in Eq. (9): the intrinsic widths of the IVGMR, $\tilde{\Gamma}_{M}^{\uparrow}$ and $\tilde{\Gamma}_{M}^{\downarrow}$, and the intrinsic spreading width of the IAS $\tilde{\Gamma}_{I A S}^{\downarrow}$, as well as the compound width $\tilde{\Gamma}_{c}$. First, the intrinsic spreading widths $\tilde{\Gamma}_{M}^{\downarrow}$ and $\tilde{\Gamma}_{I A S}^{\downarrow}$ are parametrized by means of a linear temperature dependence according to the findings of [15], 


$$
\tilde{\Gamma}^{\downarrow}=\Gamma^{\downarrow}(T=0) \cdot(1+c T),
$$

where $T$ is here the nuclear temperature in units of $\mathrm{MeV}$ and $c$ is a constant which should be determined using the results of a microscopic calculation. In general, giant resonances are described microscopically as a coherent superposition of one particle-one hole (1p-1h) excitations. The main cause of their spreading width stems from the coupling to collective low-lying surface vibrations. In Ref. [15], the spreading width of the GDR in ${ }^{120} \mathrm{Sn}$ has been studied and the slope parameter $c$ has been estimated to be about 0.025 or less, by fitting the results of microscopic calculations which take into account the coupling of the giant resonance with states composed of a $1 \mathrm{p}-1 \mathrm{~h}$ pair plus a low-lying vibration [18]. These doorway states to which the giant resonance is coupled directly, are not considered to be sharp but distributed over an energy interval of the order of $\Delta$ with a Lorentzian shape. In this spirit, the width $\Delta$ is supposed to take care of the coupling of the doorway states with the compound nucleus states. This coupling has been shown, on the basis of rather general hypothesis, to be essentially constant as a function of temperature [19]. On the other hand, we may let $\Delta$ grow when increasing $T$ to simulate the fact that the doorway states are more fragmented since the low-lying states become less collective [18]. It is found [20] that the change of the spreading width of the IAS is at most $20 \%$ when $\Delta$ is varied from $50 \mathrm{keV}$ to $500 \mathrm{keV}$, and the spreading width saturates for larger values of $\Delta$. If this $20 \%$ change of the spreading width $\tilde{\Gamma}_{I A S}^{\downarrow}$ is supposed to occur within the temperature range between 0 and $4-5 \mathrm{MeV}$, the slope parameter $c$ is $c=0.04-0.05$, which is not much different from the value mentioned above, $c=0.025$. Consequently, we take the linear dependence (11) with $c=0.025$ for $\tilde{\Gamma}_{M}^{\downarrow}$ and $\tilde{\Gamma}_{I A S}^{\downarrow}$. Then, we show below that our conclusions are not markedly changed even if we increase by a factor 2 our value of $c$.

At zero temperature, the spreading width of the IVGMR $\Gamma_{M}^{\downarrow}(T=0)$ in ${ }^{208} \mathrm{Bi}$, at the excitation energy of the IAS, is found to be of the order of $250 \mathrm{keV}$, while that of the

\footnotetext{
${ }^{1}$ This calculation was already reported in Ref. [10] where the total width of the IVGMR in ${ }^{208} \mathrm{Bi}$
} 
IAS $\Gamma_{I A S}^{\downarrow}(T=0)$ is evaluated to be about $100 \mathrm{keV} 20$ including the contribution of the charge-symmetry breaking and charge-independence breaking interactions [21]. This value $\Gamma_{I A S}^{\downarrow}(T=0)=100 \mathrm{keV}$ is in good agreement with the experimental value 17,22. Finally, $\tilde{\Gamma}_{M}^{\uparrow}(T)$ is taken as a constant according to the findings of Ref. [14] and fixed at $200 \mathrm{keV}$ ].

The compound widths $\tilde{\Gamma}_{c}$ are dominated by the most probable decay process of the compound nucleus which is usually neutron evaporation. We may discuss the neutron evaporation in simple terms, as it is done in Ref. [23] where a Weisskopf formula is used for the temperature dependence of $\tilde{\Gamma}_{n}$,

$$
\tilde{\Gamma}_{n}=\frac{2 m R^{2}}{\pi \hbar^{2}} T^{2} e^{-B_{n} / T},
$$

with a radius $R=7.1 \mathrm{fm}$ and a neutron separation energy $B_{n}=7.4 \mathrm{MeV}$ in the case of ${ }^{208} \mathrm{~Pb}$. It is known that the neutron widths obtained through this simple formula may be somewhat different from more realistic neutron widths, essentially due to the schematic assumption of a geometric absorbtion cross section for neutrons on the nucleus. In order to take into account the microscopic transmission coefficient, we have also estimated the compound widths by means of the statistical computer code CASCADE [24]. This code takes into account also proton and $\alpha$ emission and needs as an input the excitation energy $E^{*}$ of the compound system which can be related to the nuclear temperature by the wellknown relation $E^{*}=a T^{2}$ for which we have taken $a=10 \mathrm{MeV}^{-1}$. The results for the isospin mixing (9) are not so markedly sensitive to whether we use compound widths produced by CASCADE or by the simple formula (12). Therefore, for simplicity we will use the values of $\tilde{\Gamma}_{c} \equiv \tilde{\Gamma}_{n}$ obtained by using Eq. (12).

We present our results for the isospin mixing probability of ${ }^{208} \mathrm{~Pb}$ as a function of temperature in Fig. 1. All curves start, at zero temperature, from the value $\alpha^{2}=0.1$, corresponding

at the IAS energy was estimated to be about $450 \mathrm{keV}$ : about a half of the total value, that is, 250 $\mathrm{keV}$, corresponds to the spreading width whereas $200 \mathrm{keV}$ corresponds to the escape width.

\footnotetext{
${ }^{2}$ See the previous note.
} 
to a value of the spreading width of the IAS, $\Gamma_{I A S}^{\downarrow}=100 \mathrm{keV}$. The solid line corresponds to the calculation described above with the slope parameter $c=0.025$. The dashed line corresponds to choosing the parameter $c$ equal to 0.05 both for the IVGMR and for the IAS. These two results show that our general conclusions are not too much affected by the choice of $c$ and that the isospin restoration starts to take place at a temperature of about 1.5 MeV. At $3 \mathrm{MeV}$ temperature, the value of the isospin mixing probability has decreased to one fourth of the value at zero temperature due to the rapid increase of the compound width appearing in the denominator of Eq. (9)).

Experimentally, as mentioned above, the temperature dependence of the isospin mixing in the decay of the GDR was studied in the light nuclei ${ }^{28} \mathrm{Si}$ and ${ }^{60} \mathrm{Zn}[\mathbf{7}]$. A similar substantial decrease of the isospin mixing was found above $T=1 \mathrm{MeV}$ although the magnitude of the mixing at low temperature is much larger than in the present case because of the coupling to the shell model $T_{>}$states, which is more important than the coupling to the IVGMR in light nuclei [25].

In summary, we have studied the isospin mixing in compound nuclei at finite temperature with a microscopic model based on the Feshbach projection method. We are able to derive a relation which connects three physical quantities, that is, the isospin mixing amplitude, the spreading width of the IAS and the statistical decay width of the compound system. In this way, the isospin mixing probability in ${ }^{208} \mathrm{~Pb}$ as a function of the temperature is studied quantitatively for the first time by using the results of microscopic calculations. It is shown that until relatively low excitation energies, corresponding to a temperature of the order of $1 \mathrm{MeV}$, the mixing probability remains constant. At higher excitation energies, this mixing probability decreases and around $3 \mathrm{MeV}$ it is reduced by about a factor 4 because of the rapid increase of the compound width. Thus, we have verified, by using microscopic ingredients, the validity of the conjecture formulated by Wilkinson 40 years ago, of the restoration of the isospin symmetry in highly excited systems. In connection with the present work, it would be of extreme importance to obtain experimental information about the width of the IAS 
at finite temperatures.

H.S. likes to acknowledge the nice hospitality of the Università degli Studi in Milano where part of the work has been done, as well as financial support from INFN. P.F.B. and H.S. like to acknowledge the hospitality of the Institute for Nuclear Theory of the University of Washington of Seattle, where some original ideas of the present work were discussed and stimulating discussions with K.A. Snover took place. 


\section{REFERENCES}

[1] W. Heisenberg, Z. Phys. 77 (1932) 1.

[2] J.D. Anderson and C. Wong, Phys. Rev. Lett. 7 (1961) 250; ibid. 8 (1962) 442;

J.D. Anderson, C. Wong and J.W. McClure, Phys. Rev. 126 (1962) 2170.

[3] A.C. Mueller and B.M. Sherrill, Ann. Rev. Nucl. Part. Sci. 43 (1993) 529; I. Tanihata, Progr. Nucl. Part. Phys. 35 (1995) 505.

[4] I. Hamamoto and H. Sagawa, Phys. Rev. C 48 (1993) R960; G. Colò, M.A. Nagarajan, P. Van Isacker and A. Vitturi, Phys. Rev. C 52 (1995) R1175.

[5] H. Morinaga, Phys. Rev. 97 (1955) 444.

[6] D.H. Wilkinson, Philos. Mag. 1 (1956) 379.

[7] M.N. Harakeh et al., Phys. Lett. B 176 (1986) 297; J.A. Behr et al., Phys. Rev. Lett. 70 (1993) 3201; K.A. Snover, Nucl. Phys. A 553 (1993) 153c.

[8] M. Cinausero et al., Z. Phys. A 352 (1995) 273.

[9] V.V. Sokolov and V. Zelevinsky, Phys. Rev. C 56 (1997) 311.

[10] T. Suzuki, H. Sagawa, G. Colò, Phys. Rev. C 54 (1996) 2954.

[11] N. Auerbach, Phys. Rep. 98 (1983) 273.

[12] Cf., e.g., D.M. Landau and E.M. Lifshitz, Course of Theoretical Physics, Vol. 4, Relativistic Quantum Theory (Pergamon Press, 1971), pp. 203-207.

[13] J.J. Gaardhøje, Ann. Rev. Nucl. Part. Sci. 42 (1992) 488.

[14] H. Sagawa and G.F. Bertsch, Phys. Lett. B 146 (1984) 138.

[15] P. Donati, N. Giovanardi. P.F. Bortignon, R.A. Broglia, Phys. Lett. B 383 (1996) 15 and Refs. therein. 
[16] P.F. Bortignon, A. Bracco, D. Brink and R.A. Broglia, Phys. Rev. Lett. 67 (1991) 3360.

[17] H.L. Harney, A. Richter and H.A. Weidenmüller, Rev. Mod. Phys. 58 (1986) 607.

[18] P.F. Bortignon, R.A. Broglia, G.F. Bertsch, J. Pacheco, Nucl. Phys. A 460 (1986) 149.

[19] B. Lauritzen, P.F. Bortignon, R.A. Broglia and V.G. Zelevinsky, Phys. Rev. Lett. 74 (1995) 5190.

[20] G. Colò, H. Sagawa, N. Van Giai, P.F. Bortignon and T. Suzuki, Phys. Rev. C 57 (1998) 3049.

[21] H. Sagawa, N. Van Giai and T. Suzuki, Phys. Rev. C 53 (1996) 2163; H. Sagawa, N. Van Giai and T. Suzuki, Phys. Lett. B 353 (1995) 7.

[22] H. Orihara et al., to be published.

[23] D.M. Brink, Nucl. Phys. A 519 (1990) 3c.

[24] F. Pulhofer, Nucl. Phys. A 280 (1977) 267.

[25] A.Z. Mekjian, Phys. Rev. Lett. 25 (1970) 888. 


\section{Fig.1}

The temperature dependence of the isospin mixing probability $\alpha^{2}$ in ${ }^{208} \mathrm{Bi}$. This probability is calclulated by using Eq. (9), and assuming a linear temperature dependence for $\tilde{\Gamma}_{M}^{\downarrow}$ and $\tilde{\Gamma}_{I A S}^{\uparrow}$ according to Eq. (11). The slope parameters $c$ adopted in (11) are 0.025, and 0.05 for the solid and dashed curves, respectively. The value of $\tilde{\Gamma}_{c}$ is evaluated through Eq. (12). 


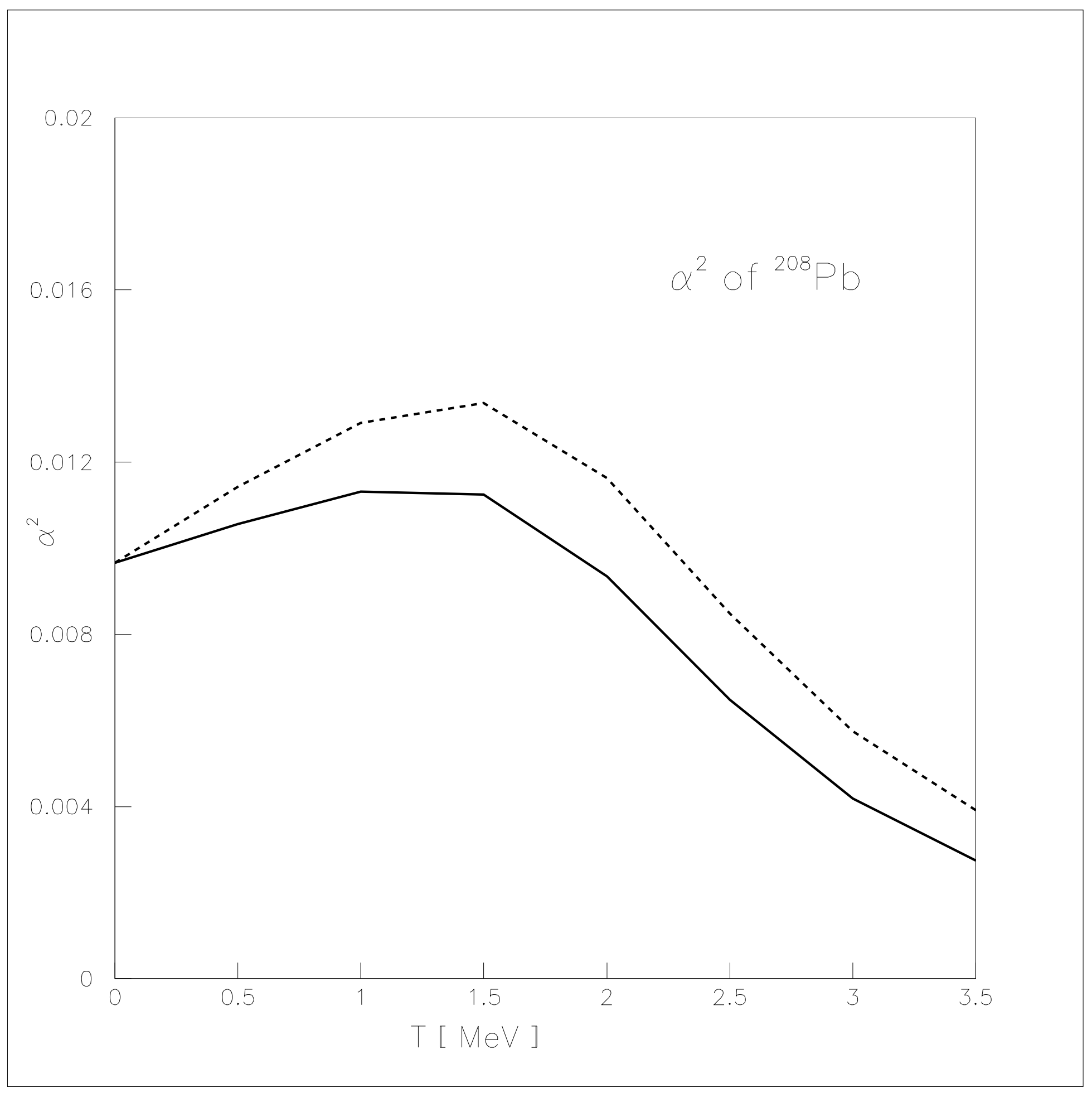

